\title{
Educação Remota Emergencial (ERE): Um estudo empírico sobre Capacidades Educacionais e Expectativas Docentes durante a Pandemia da COVID-19
}

\author{
Emergency Remote Education (ERE): An empirical study on Educational Capabilities and \\ Teaching Expectations during the COVID-19 Pandemic
}

Enseñanza Remota de Emergencia (ERE): Un Estudio Empírico sobre Capacidades Educativas y

Expectativas Docentes durante la Pandemia de la COVID-19

Recebido: 12/01/2021 | Revisado: 14/01/2021 | Aceito: 15/01/2021 | Publicado: 18/01/2021

Gustavo Henrique Silva de Souza
ORCID: https://orcid.org/0000-0002-4046-9669
Instituto Federal de Educação, Ciência e Tecnologia do Norte de Minas Gerais, Brasil
E-mail: gustavo.souza@ @ifnmg.edu.br
Wallas Siqueira Jardim
ORCID: https://orcid.org/0000-0002-8740-0482
E-mail: wallas.jardim@ifnmg.edu.br
Yuri Bento Marques
Instituto Federal de Educação, Ciência e Tecnologia do Norte de Minas Gerais, Brasil
ORCID: https://orcid.org/0000-0003-3414-2325
E-mail: yurimarques@ @mail.com
Geraldo Lopes Junior
Instituto Federal de Educação, Ciência e Tecnologia do Norte de Minas Gerais, Brasil
ORCID: https://orcid.org/0000-0002-2243-2336
E-mail: geraldo.lopes@ifnmg.edu.br
Aline Patrícia Sobral dos Santos
Instituto Federal de Educação, Ciência e Tecnologia do Norte de Minas Gerais, Brasil
ORCID: https://orcid.org/0000-0002-7065-6959
Centro Universitário de Maringá, Brasil
E-mail: aline.filo.edu@gmail.com
Leandro de Paula Liberato
ORCID: https://orcid.org/0000-0002-2778-0575
E-mail: leandro.liberato@ @ifnmg.edu.br

\section{Resumo}

Este estudo teve por objetivo identificar as condições, expectativas e capacidades de professores de cursos presenciais para a realização de atividades de ensino (letivas) remotas mediadas por tecnologias digitais, em virtude do distanciamento social associado à pandemia da COVID-19. Desenvolveu-se uma pesquisa exploratória, de abordagem quantitativa, com uma amostra não-probabilística de conveniência de 588 professores brasileiros de instituições públicas e privadas de ensino presencial de diversas áreas do conhecimento. Os participantes foram convidados pelas redes sociais a responder voluntariamente a um questionário on-line, entre os dias 19 e 30 de junho de 2020. Foram realizadas análises estatísticas descritivas de parametrização simples (p.ex., frequência, média, desvio-padrão), bem como análises inferenciais, por exemplo, a análise fatorial exploratória e a regressão linear múltipla. Os resultados destacam que a maioria dos professores investigados apresentou predisposição e interesse em realizar atividades remotas, além de condições técnicas de acesso e uso de internet e de equipamentos digitais e computacionais. Apesar disso, a instituições escolares precisam se atentar os intervenientes associados ao ambiente domiciliar, como as demandas domésticas e familiares e as necessidades individuais dos professores que podem se tornar barreiras para o efetivo desenvolvimento da Educação Remota Emergencial.

Palavras-chave: Remota emergencial; Docentes; Ensino superior; Ensino técnico; COVID-19.

\begin{abstract}
This study aimed to identify the conditions, expectations, and capacities of professors from face-to-face courses (technical and higher education) to carry out remote teaching activities mediated by digital technologies, due to the COVID-19 pandemic and the social distancing measures. Exploratory and quantitative research was developed, with a convenience non-probabilistic sample of 588 Brazilian professors from public and private educational institutions. Participants were invited by social networks to complete voluntarily an online questionnaire, between June 19 and 30 ,
\end{abstract}


2020. Descriptive statistical analyses were performed (e.g., frequency, mean, standard deviation), as well as inferential analyses, for example, exploratory factor analysis and multiple linear regressions. The results highlight that the majority of the investigated professors showed a predisposition and interest in performing remote teaching activities, in addition to technical conditions for accessing and using the internet and digital and computational equipment. Despite this, school institutions need to pay attention to the factors associated with the home environment, such as domestic and family demands and the individual needs who can become barriers to the effective development of Emergency Remote Education.

Keywords: Emergency remote education; Professors; Higher education; Technical education; COVID-19.

\section{Resumen}

Este estudio tuvo como objetivo identificar las condiciones, expectativas y capacidades de los docentes de los cursos presenciales para el desempeño de actividades de enseñanza a distancia mediadas por tecnologías digitales, debido a la distancia social asociada a la pandemia de la COVID-19. Se realizó una investigación exploratoria, con enfoque cuantitativo, con una muestra no probabilística de conveniencia de 588 docentes brasileños de instituciones públicas y privadas de docencia presencial de diferentes áreas del conocimiento. Los participantes fueron invitados por las redes sociales a responder voluntariamente a un cuestionario en línea, entre el 19 y 30 de junio de 2020. Se realizaron análisis estadísticos descriptivos de parametrización simple (ej., frecuencia, media, desviación estándar), así como análisis inferenciales, por ejemplo, análisis factorial exploratorio y regresión lineal múltiple. Los resultados resaltan que la mayoría de los docentes investigados mostraron predisposición e interés por realizar actividades a distancia, además de condiciones técnicas para el acceso y uso de internet y equipos digitales y computacionales. A pesar de ello, las instituciones escolares deben prestar atención a los actores asociados al entorno del hogar, como las demandas domésticas y familiares y las necesidades individuales de los docentes, que pueden convertirse en barreras para el desarrollo efectivo de la Enseñanza Remota de Emergencia.

Palabras clave: Enseñanza remota de emergencia; Profesores; Enseñanza superior; Educación técnica; COVID-19.

\section{Introdução}

Com a deflagração da pandemia associada à COVID-19 (Novo Coronavírus), diversas medidas de mitigação e supressão do contágio da doença foram tomadas no mundo inteiro, em especial, o distanciamento social (Anderson, Heesterbeek, Klinkenberg, \& Hollingsworth, 2020). O distanciamento social visa à diminuição de interação e proximidade entre as pessoas em locais onde a transmissão possa ocorrer em larga escala, compreendendo a permanência obrigatória em casa e a adoção do trabalho remoto (home office), em virtude do fechamento, por exemplo, dos comércios e serviços nãoessenciais e das instituições de ensino (escolas, faculdades etc.) (Bedford, et al., 2020; Remuzzi \& Remuzzi, 2020; Viner, et al., 2020).

Além disso, uma vez que os impactos da COVID-19 ao médio e longo prazo ainda são incertos (Peeri, et al., 2020) e o Brasil caminha para se tornar o epicentro da doença no mundo, com cerca de 3.532.330 casos e 113.358 mortes entre fevereiro e agosto de 2020 (World Health Organization [WHO], 2020), as medidas restritivas passam a ser obrigatórias por um período mais prolongado e intermitente (Kissler, Tedijanto, Goldstein, Grad, \& Lipsitch, 2020).

Especificamente, no caso do Brasil, o fechamento das instituições de ensino e a suspensão das aulas presenciais foram normatizados pelas Portarias 343/2020, 345/2020, 376/2020 e 395/2020 do governo federal (Brasil, 2020a, 2020b, 2020c, 2020d), que autorizaram em caráter excepcional, a partir de 18 de março até 31 de dezembro de 2020, a substituição das aulas presenciais por aulas ministradas remotamente por meios digitais - considerando a legislação em vigor e a validade das atividades remotas como dias letivos, sem a necessidade de alteração dos Projetos Pedagógicos de Curso (PPCs).

Apesar disso, segundo o Ministério da Educação (Brasil, 2020e), a maioria das instituições de ensino permaneceu sem atividades letivas (mesmo após cinco meses da portaria de autorização das aulas remotas), dada às necessidades de planejamento institucional e organização escolar quanto às demandas didático-pedagógicas.

No entanto, diante da pressão por uma alternativa às aulas presenciais suspensas, as dificuldades do retorno das atividades letivas remotamente recaem sobre os dois principais atores que estão na linha de frente deste processo: estudantes e professores. Se, por um lado, condições técnicas (acesso à internet e equipamentos) são essenciais para a realização de atividades remotas. Por outro lado, aspectos sociais, familiares e psicológicos se mostram balizadores do aproveitamento e do 
desempenho efetivos relacionados a essas atividades - conforme experiências de cenários epidêmicos anteriores (ver, Taylor, 2019).

Uma vez que estudos sobre as expectativas de estudantes frente às possibilidades aulas remotas durante o distanciamento social já vêm sendo desenvolvidos (Souza, et al., 2020), este trabalho segue para outro viés investigativo e tem por objetivo identificar as condições, expectativas e capacidades de professores de cursos técnicos e superiores presenciais para a realização de atividades de ensino (letivas) remotas mediadas por tecnologias digitais, em virtude do distanciamento social associado à pandemia da COVID-19.

Este estudo emerge do seguinte problema de pesquisa: professores prioritariamente de cursos presenciais apresentam condições para desenvolver atividades letivas remotas e possuem competências docentes específicas necessárias para este fim, dadas as similaridades ferramentais e metodológicas com a Educação a Distância (EaD)?

Discussões recentes (Barreto \& Rocha, 2020; Macedo, Ornellas, \& Bomfim, 2020) alertam que as articulações políticas para a realização (leia-se: imposição) de atividades letivas remotas são negligentes às idiossincrasias da escola presencial, incluindo suas capacidades técnicas, seus docentes e o pessoal de apoio pedagógico - confluindo, talvez, em práticas educacionais insipientes.

\section{Possibilidades da Educação Remota Emergencial (ERE)}

Consensualmente, as possibilidades de retorno das atividades letivas por meio de aulas/estudos remotos e tecnologias digitais de comunicação e informação (TDICs) têm sido consideradas globalmente (Toquero, 2020; Williamson, Eynon, \& Potter, 2020), sob o uso das expressões Educação Remota Emergencial (ERE ou, em inglês, Emergency Remote Education) e Ensino Remoto Emergencial (ERE ou, em inglês, Emergency Remote Teaching).

Em termos conceituais, torna-se necessário distinguir terminologias específicas, visando evitar ambiguidades interpretativas, no que tange às diferentes implicações da Educação à Distância $(\mathrm{EaD})$ e da Educação Remota Emergencial (ERE). A EaD é uma modalidade educacional que considera um planejamento pedagógico de médio e longo prazo, com suporte tutorado em ambientes virtuais de aprendizagem (AVA) e plataformas específicas de comunicação, em que a aprendizagem ocorre de maneira autônoma. $\mathrm{Na} \mathrm{EaD}$, o desenvolvimento do processo de ensino-aprendizagem prevê o uso de material didático próprio inserido em um escopo lógico e de qualidade estética, bem como docentes, tutores e apoio pedagógico previamente treinados e capacitados (Beluce \& Oliveira, 2016; Coelho \& Tedesco, 2017; Maia \& Mattar, 2008; Nunes, Oliveira, \& Sabino, 2019).

Por outro lado, a ERE - cujos termos "estudos remotos", "educação remota", "ensino remoto" podem ser utilizados como sinônimos, em virtude da proximidade semântica - se configura como uma adaptação de técnicas e ferramentas didáticopedagógicas que se utilizam de metodologias da $\mathrm{EaD}$, instruções para estudos orientados e autônomos, e atividades de ensinoaprendizagem não-presenciais mediadas por TDICs, além de interações síncronas e assíncronas para a resolução de dúvidas ou a oferta de conteúdos curriculares por meio de mídias sociais digitais e a disponibilização prévia de material didático e acadêmico (no formato impresso ou digital) (Arruda, 2020; Joye, Moreira, \& Rocha, 2020; Williamson, Eynon, \& Potter, 2020). Williamson, Eynon e Potter (2020) acreditam que as práticas didático-pedagógicas vinculadas à ERE serão protótipos para uma mudança de paradigma na educação, para além da pandemia da COVID-19.

Essa linha de pensamento indica que os docentes da educação presencial precisarão ressignificar as suas práticas didático-pedagógicas para atuar com proficiência na ERE, submetendo-se a modelos baseados em metodologias ativas de ensino, além do uso de TDICs e de ferramentas da EaD.

Especificamente na EaD, o professor deve ter domínio das TDICs para compartilhar conhecimentos objetivos e adequados por meio de vídeos e textos, com competências para manter e incentivar relações interpessoais digitalmente e 
gerenciamento de grupos e discussões on-line, capacidade para se comunicar de forma clara, inteligível, compreensiva e empática, e apresentação de uma postura ético-dialógica (Mill, 2012; Nunes, Oliveira, \& Sabino, 2019).

Diferentemente dos nativos digitais - aqueles que nasceram após os anos 2000, inseridos em um cenário digital e tecnológico comum (Azevedo, Silveira, Lopes, Amaral, Goulart, \& Martins, 2018; Freitas, 2010) -, que é a grande maioria dos estudantes, muito professores estão ainda aprendendo a dominar as TDICs e se inserindo na cibercultura, que permeia novas possibilidades de colaboração e atuação educacional. Kaieski, Grings e Fetter (2015), por exemplo, analisam propostas didático-pedagógicas por meio do uso do Whatsapp, incluindo nesse âmbito os grupos sociais, a possibilidade de discussões e o compartilhamento e troca de materiais - visando a aproximação dos próprios estudantes entre si, bem como dos estudantes com professores e escola.

Essa discussão ocorre porque, diferente da educação presencial, na EaD e também na ERE, o estudante é um agente autônomo da própria aprendizagem, estabelecendo o seu próprio ritmo individual de leituras, estudos e reflexões. Este é um desafio iminente para a efetividade do processo de ensino remoto (Castaman \& Rodrigues, 2020).

Embora seja imprescindível que o estudante trace suas próprias metas e objetivos mensuráveis de estudo, vale ressaltar que estudar à distância ou remotamente não é necessariamente aprender sozinho, mas sim, uma aprendizagem cujo ambiente de compartilhamento de conhecimento ultrapassa as fronteiras de tempo e espaço, redimensionando a sala de aula, a biblioteca, os laboratórios etc. (Camacho, Joaquim, Menezes, \& Sant'Anna, 2020; Gomes, et al., 2020).

Nesse aspecto, a posição de comprometimento e responsabilidade com a aprendizagem é o cerne da ERE, em que não apenas os materiais, os vídeos, os conteúdos ou as ferramentas serão determinantes em si, mas a maturidade do estudante, do professor e da escola em busca por uma nova forma de fazer o processo educacional (Arruda, 2020).

Além disso, mais do que condições técnicas (como acesso à internet ou a equipamentos), a ERE poderá exigir uma série de atividades administrativas exaustivas aos professores e equipes pedagógicas em virtude da natureza do distanciamento, conforme pode ser visto nas propostas de educação remota, por exemplo. Isso ocorre porque a ERE foi planejada sob um formato burocrático e vertical, sob a justificativa de se adaptar as aulas presenciais temporariamente (Saraiva \& Traversini; Lockmann, 2020).

Assim, em termos de trabalho docente, a ERE compreende múltiplas atividades de diferentes ordens, por exemplo: ajustar os conteúdos programáticos, preparar planos de aula, materiais e atividades, preencher relatórios e planilhas, participar de reuniões on-line, realizar encontros ou aulas on-line com os estudantes, corrigir trabalhos e lançar dados no sistema escolar, dentro do ambiente domiciliar, concomitantemente à realização de serviços e atividades domésticos, além do suporte afetivo e estrutural a filhos, parentes e cônjuges (em especial, aqueles que exigem cuidado intermitente).

Sobre este tema, Sanchez-Taltavull, Candinas, Roldán e Beldi (2020) ressaltam que a produtividade no trabalho durante o distanciamento social pode diminuir significativamente, especialmente quando não há um ambiente adequado para o trabalho remoto ou quando há altas taxas de infecção por COVID-19 na localidade - estimando uma redução de 50\% na produtividade normal. Alguns autores (Barros \& Silva, 2010; Bathini \& Kandathil, 2019; Christensen, 1988; Gaudecker, Holler, Janys, Siflinger, \& Zimpelmann, 2020) destacam elementos condicionantes para uma melhor atuação no trabalho remoto (home office), por exemplo: (i) capacidade de autonomia e autodisciplina, (ii) condições de acesso aos colegas de trabalho, (iii) conhecimento básico dos recursos e procedimentos a serem, respectivamente, utilizados e realizados, (iv) natureza das atividades e tarefas de trabalho, (v) quantidade de pessoas dentro de casa e incidência de crianças pequenas ou pessoas com necessidades especiais, (vi) espaço domiciliar adequado.

Frente ao desafio educacional e gerencial subjacente, a discussão sobre as possibilidades de implementação da ERE se torna essencial. Assim, ao passo que se torna necessário capacitar a escola presencial para a educação remota, as pesquisas sobre ERE podem contribuir para fornecer uma orientação prática e fundamentalmente adequada para as propostas didático- 
pedagógicas que se fazem necessárias.

\section{Métodos}

\subsection{Tipo de pesquisa}

Em termos metodológicos, este trabalho apresenta um delineamento exploratório na maneira de condução da pesquisa e de abordagem quantitativa no modo de análise, em virtude do objetivo do estudo. A pesquisa exploratória associada a modelos quantitativos de análise tem, em seu escopo, trata-se de uma investigação inicial que visa compreender um fenômeno ainda pouco explorado e com necessidade de aprofundamento teórico-empírico (Pereira, Shitsuka, Parreira, \& Shitsuka, 2018).

\subsection{Participantes e procedimentos}

Participaram do estudo 588 professores brasileiros (52,4\% do sexo feminino), com idade variando de 24 a 69 anos $\left(\mathrm{M}_{\text {idade }}=40,15 ; \mathrm{DP}_{\text {idade }}=8,32\right)$, dos quais $72,8 \%$ de instituições públicas estaduais e federais de ensino (médio, técnico e superior) e $27,2 \%$ de instituições privadas de ensino presenciais de diversas áreas do conhecimento. Os participantes foram oriundos de 12 (doze) diferentes Estados federativos brasileiros: Minas Gerais (94,3\%), Bahia (1,2\%), São Paulo (1\%), Alagoas $(0,7 \%)$, Goiás $(0,6 \%)$, Rio Grande do Sul $(0,5 \%)$, Rio de Janeiro $(0,4 \%)$, Mato Grosso $(0,4 \%)$, Piauí $(0,3 \%)$, Ceará $(0,2 \%)$, Espírito Santo $(0,2 \%)$ e Paraná $(0,2 \%)$. Dos participantes, cinco $(0,9 \%)$ professores relataram suspeita de contágio com COVID-19 (Novo Coronavírus) e 70 (11,9\%) relataram suspeita ou confirmação entre parentes e amigos próximos de contágio com COVID-19 (Novo Coronavírus).

O procedimento amostral foi não-probabilístico, com coleta de dados por acessibilidade e de forma individual, em que os professores foram convidados pelas redes sociais a responder voluntariamente ao questionário on-line, entre os dias 19 e 30 de junho de 2020. Os participantes foram esclarecidos sobre o estudo em questão e acerca do anonimato e sigilo de suas respostas, sendo requerida a assinatura de um Termo de Consentimento Livre e Esclarecido, garantindo o caráter voluntário da participação e respeito às diretrizes éticas que regem a pesquisa com seres humanos (CAAE: 31508720.8.0000.8747). Em média, 12 minutos foram demandados para se concluir a participação no estudo.

\subsection{Instrumentos}

Para a realização deste estudo, foram desenvolvidos quatro novos instrumentos e um questionário sociodemográfico. Inicialmente, foi realizado um brainstorming para compreender as questões a serem mensuradas. Em virtude da excepcionalidade da temática em estudo e da falta de instrumentos prontos a serem utilizados, os questionários foram planejados (54 itens ao todo) de modo a mapear as condições de retorno das atividades escolares remotamente no Brasil na perspectiva docente. Os questionários foram desenvolvidos de acordo com os critérios psicométricos estabelecidos por Cohen e Swerdlik (2017). Os questionários foram submetidos ao procedimento de análise de conteúdo (adequação dos itens à proposta da pesquisa) e à análise semântica (inteligibilidade dos itens). A validade de conteúdo foi realizada com oito juízes (sendo três psicometristas e cinco professores das áreas de línguas, geografia, matemática, informática e administração de empresas). A validade semântica foi realizada com sete juízes (sendo dois psicometristas, dois professores da área de línguas e três alunos). Como resultado, nove itens foram excluídos. Os questionários desenvolvidos seguem descritos a seguir:

(i) Escala de Reações Prospectivas frente ao Trabalho Remoto Docente (ERP-TRD): questionário baseado em uma escala de 5 pontos contínuos (variando de $1=$ Discordo Totalmente a $5=$ Concordo Totalmente). O ERP-TRD é composto por 16 itens, relacionados à suspensão das aulas, às possibilidades de trabalho/ensino remoto por meio de TDICs e às experiências cotidianas durante o período de distanciamento social, como: "gostaria que a Instituição em que eu leciono ofertasse aulas à distância para os discentes de regime presencial durante o distanciamento social", "sinto-me motivado a continuar o trabalho 
remotamente (à distância)", e "tenho dúvidas sobre a efetividade de avaliações (testes / provas) ou acompanhamento nas atividades à distância (on-line)".

(ii) Escala de Capacidades Educacionais Inovadoras (ECEI): questionário baseado em uma escala de 5 pontos contínuos (variando de 1 = Discordo Totalmente a 5 = Concordo Totalmente), contendo 13 itens, por exemplo: "Tenho conhecimento de metodologias inovadoras de ensino relacionadas às tecnologias digitais de informação e comunicação" e "Mantenho-me informado sobre orientações educacionais mais atualizadas".

(iii) Questionário de Condições para o Uso de Tecnologias Digitais de Informação e Comunicação (QCU-TDIC): verificou a efetividade do trabalho/ensino remoto por meio das condições de acesso e uso de tecnologias digitais. O questionário se baseia em uma escala discreta de 5 pontos, composto por 9 itens, por exemplo: "Tempo diário disponível para realizar atividades à distância (fazer leituras e trabalhos, responder fóruns etc.)" e "Habilidade com uso de ferramentas computacionais (editores de texto, planilhas, apresentações, navegação web etc.)".

(iv) Questionário de Habilidades com Aplicativos de Comunicação, Compartilhamento e Videoconferência (QHA-7): verificou a proximidade (e conhecimento) com ferramentas que podem ser utilizadas no trabalho/ensino remoto, a partir das expectativas de uso de aplicativos. O questionário se baseia em uma escala de 5 pontos contínuos (variando de 1 = Não conhece a $5=$ Conhece e possui expertise ou grande habilidade), composto por 7 itens, por exemplo: "Google Drive", "Zoom" e "Hangouts Meets".

(v) Questionário Sociodemográfico: visou conhecer e caracterizar a amostra. Este questionário complementar contou com questões, como: gênero, idade, localidade, suspeitas ou confirmações de casos de COVID-19, dentre outras.

\subsection{Análises}

Inicialmente, os dados foram processados e analisados por meio de estatísticas descritivas de parametrização simples (p.ex., frequência, percentual, média, desvio-padrão). Com o objetivo de encontrar o conjunto mais parcimonioso de elementos que explicam os constructos avaliados, ajuizou-se o uso da análise fatorial exploratória.

Os instrumentos ERP-TRD e ECEI, por apresentarem uma operacionalização pautada em um escalonamento contínuo e métodos de desenvolvimento dos itens baseados em parâmetros psicométricos, foram submetidos à análise fatorial exploratória com método de extração a máxima verossimilhança, utilizando a rotação varimax com a padronização Kaiser. Por sua vez, os instrumentos QCU-TDIC e QHA-7, por apresentarem uma operacionalização em escala discreta, foram submetidos à análise de componentes principais, cujo objetivo é otimizar a variância dos itens, de modo a determinar uma explicação pautada no componente que agrega maior proporção da variação total dos itens. Foram utilizados os critérios de significância estatística (cargas fatoriais acima de $|0,300|$ ), autovalores acima de 1,0 e alfa de Cronbach (teste de confiabilidade) acima de 0,600 (Hair, et al., 2010).

Em complemento, visando testar um modelo explicativo de possíveis antecedentes (variáveis independentes) aos aspectos analisados e relacionados especificamente ao trabalho remoto (variável dependente) - neste caso, fatores da ERPTRD -, utilizou-se a regressão linear múltipla (método stepwise), considerando a matriz de covariâncias e avaliando a homocedasticidade e a multicolinearidade entre as variáveis preditoras, dentro dos parâmetros de validação do modelo recomendados por Hair et al. (2010).

\section{Resultados}

\subsection{Expectativas sobre o trabalho remoto docente no período de distanciamento social}

Inicialmente, apresentam-se os resultados descritivos da Escala de Reações Prospectivas frente ao Trabalho Remoto Docente (ERP-TRD), na Tabela 1. Os resultados mostram a prevalência pelo interesse e disponibilidade em trabalhar 
remotamente (itens 1, 7 e 16), em participar de cursos de capacitação (item 6) e utilizar as redes sociais como mecanismo de envolvimento com colegas e estudantes (item 5). Apesar disso, embora 39,3\% dos docentes alegaram que não gostariam que a Instituição em que leciona ofertasse aulas remotas para os discentes de regime presencial durante o distanciamento social (item 4), de modo geral, os itens com menor endosso que podem prejudicar o processo de ERE seria a falta de experiência dos docentes em ambientes virtuais de aprendizagem (itens 14 e 15).

Tabela 1: Expectativas Docentes sobre o trabalho remoto no período de distanciamento social.

\begin{tabular}{|c|c|c|c|c|c|}
\hline \multirow{2}{*}{ Itens } & \multirow{2}{*}{ Média } & \multirow{2}{*}{ DP } & \multicolumn{3}{|c|}{ Endosso ao item } \\
\hline & & & Baixo & Médio & Alto \\
\hline $\begin{array}{l}\text { 6. Interesso-me em participar de cursos de formação para atuar na } \\
\text { Educação à Distância. }\end{array}$ & 4,26 & 1,05 & $7,8 \%$ & $11,6 \%$ & $80,6 \%$ \\
\hline $\begin{array}{l}\text { 16. Independente da suspensão do calendário letivo, eu continuo } \\
\text { trabalhando durante esse período de distanciamento social. }\end{array}$ & 4,15 & 1,17 & $10,7 \%$ & $13,3 \%$ & $76,0 \%$ \\
\hline $\begin{array}{l}\text { Interesso-me por participar de fóruns/grupos de discussão com os } \\
\text { olegas sobre assuntos especificamente de trabalho. }\end{array}$ & 4,06 & 1,07 & $9,2 \%$ & $6,2 \%$ & $74,7 \%$ \\
\hline $\begin{array}{l}\text { 5. Acredito que as redes sociais podem ser utilizadas para que } \\
\text { possamos compartilhar informações com os discentes e servidores }\end{array}$ & 77 & 1,26 & $17,7 \%$ & $7,5 \%$ & $64,8 \%$ \\
\hline $\begin{array}{l}\text { a efetividade de avaliações (testes / provas) } \\
\text { s atividades à distância (on-line). }\end{array}$ & 3,49 & 1,32 & $22,6 \%$ & $24,8 \%$ & $52,6 \%$ \\
\hline $\begin{array}{l}\text { cia como discente em Ambientes Virtuais de } \\
\text { A). }\end{array}$ & 3,34 & 1,59 & $\%$ & $\%$ & $\%$ \\
\hline $\begin{array}{l}\text { ando a suspensão do calendário letivo, pra estimular } \\
\text { ssoais. }\end{array}$ & 3,25 & 1,27 & $26,7 \%$ & $29,8 \%$ & $43,5 \%$ \\
\hline $\begin{array}{l}\text { nsão do calendário letivo não é algo bom para } \\
\text { issionais e acadêmicos. }\end{array}$ & 3,22 & 1,38 & $29,8 \%$ & $25,3 \%$ & $44,9 \%$ \\
\hline vado a continuar o trabalho remotamente (à & 3,22 & 1,20 &, $7 \%$ & $4,7 \%$ & $39,6 \%$ \\
\hline $\begin{array}{l}\text { 8. Sinto que eu tenho dificuldade na realização das minhas atividades } \\
\text { à distância. }\end{array}$ & 3,03 & 1,33 & $35,7 \%$ & $24,0 \%$ & $40,3 \%$ \\
\hline $\begin{array}{l}\text { 4. Gostaria que a Instituição em que leciono ofertasse aulas à } \\
\text { distância para os discentes de regime presencial durante o } \\
\text { distanciamento social. }\end{array}$ & 2,98 & 1,51 & $39,3 \%$ & $19,9 \%$ & $40,8 \%$ \\
\hline $\begin{array}{l}\text { 11. O ambiente em casa não me permite ter concentração para } \\
\text { realizar trabalhos remotos. }\end{array}$ & 2,67 & 1,38 & $46,1 \%$ & $24,8 \%$ & $29,1 \%$ \\
\hline $\begin{array}{l}\text { 10. Tenho dificuldade de leitura de livros (e-book ou PDF) e textos } \\
\text { pela tela do computador. }\end{array}$ & 2,55 & 1,39 & $52,6 \%$ & $20,6 \%$ & $26,9 \%$ \\
\hline $\begin{array}{l}\text { 14. Tenho experiência como docente em Ambientes Virtuais de } \\
\text { Aprendizagem (AVA). }\end{array}$ & 2,48 & 1,57 & $57,5 \%$ & $12,8 \%$ & $29,8 \%$ \\
\hline $\begin{array}{l}\text { 12. Meus compromissos domésticos ou familiares me impedem de } \\
\text { realizar quaisquer outras atividades. }\end{array}$ & 2,16 & 1,17 & $63,9 \%$ & $20,6 \%$ & $15,5 \%$ \\
\hline $\begin{array}{l}\text { 15. Tenho experiência como tutor em Ambientes Virtuais de } \\
\text { Aprendizagem (AVA). }\end{array}$ & 2,07 & 1,53 & $70,1 \%$ & $7,7 \%$ & $22,3 \%$ \\
\hline
\end{tabular}

Fonte: Autores.

Ademais, vale destacar que alguns dos itens de menor endosso, como as demandas domésticas e familiares (item 12), a concentração no ambiente domiciliar (item 11) e a dificuldade de leitura de livros e textos pela tela do computador (item 10), embora demonstrem baixa influência ou prejuízo ao processo de ERE, aqueles docentes que alegam sofrer mais contundentemente com essas questões poderão ser impedidos de realizar as atividades letivas remotamente. Um olhar detalhado mostra que o endosso a esses itens críticos não pode ser tratado de maneira insignificante - sob o risco de realizar planejamentos didático-pedagógicos insipientes.

Por conseguinte, visando demonstrar uma explicação parcimoniosa ao conjunto de itens analisados, ajuizou-se o uso da análise fatorial exploratória (AFE). Verificada a adequação dos dados e a fatoriabilidade da matriz de correlações dos itens 
da Escala de Reações Prospectivas ao Trabalho Remoto Docente (ERP-TRD), por meio das estatísticas de Kaiser-Meyer-Olkin $($ KMO $)=0,788$ e do Teste de Esfericidade de Bartlett, $\chi^{2}(120)=2192,861 ; p=0,000$; procedeu-se à AFE, utilizando o método de extração de máxima verossimilhança e rotação Varimax com normatização de Kaiser, convergida em 6 iterações. Foram extraídos 4 (quatro) fatores (Tabela 2), com uma explicação de 38,10\% da variância total e cargas fatoriais que variaram de $|0,302|$ a $|0,793|$.

Tabela 2: Cargas Fatoriais, Consistência Interna e Extração dos Fatores da ERP-TRD.

\begin{tabular}{|c|c|c|c|c|}
\hline \multirow{2}{*}{ Itens } & \multicolumn{4}{|c|}{ Fatores } \\
\hline & 1 & 2 & 3 & 4 \\
\hline $\begin{array}{l}\text { 8. Sinto que eu tenho dificuldade na realização das minhas } \\
\text { atividades docentes de forma remota. }\end{array}$ & 0,757 & & & \\
\hline $\begin{array}{l}\text { 9. Tenho dúvidas sobre a efetividade de avaliações (testes / } \\
\text { provas) ou acompanhamento nas atividades à distância (on-line). }\end{array}$ & 0,569 & & & \\
\hline $\begin{array}{l}\text { 10. Tenho dificuldade de leitura de livros (e-book ou PDF) e } \\
\text { textos pela tela do computador. }\end{array}$ & 0,424 & & & \\
\hline $\begin{array}{l}\text { 4. Gostaria que a Instituição em que eu leciono ofertasse aulas } \\
\text { remotas aos discentes de regime presencial durante o } \\
\text { distanciamento social. }\end{array}$ & $-0,361$ & & & \\
\hline $\begin{array}{l}\text { 14. Tenho experiência como docente em Ambientes Virtuais de } \\
\text { Aprendizagem (AVA). }\end{array}$ & & 0,752 & & \\
\hline $\begin{array}{l}\text { 15. Tenho experiência como tutor em Ambientes Virtuais de } \\
\text { Aprendizagem (AVA). }\end{array}$ & & 0,705 & & \\
\hline $\begin{array}{l}\text { 13. Tenho experiência como discente em Ambientes Virtuais de } \\
\text { Aprendizagem (AVA). }\end{array}$ & & 0,468 & & \\
\hline $\begin{array}{l}\text { 12. Meus compromissos domésticos ou familiares me impedem } \\
\text { de realizar quaisquer outras atividades. }\end{array}$ & & & 0,785 & \\
\hline $\begin{array}{l}\text { 11. O ambiente em casa não me permite ter concentração para } \\
\text { realizar trabalhos remotos. }\end{array}$ & & & 0,716 & \\
\hline $\begin{array}{l}\text { 1. Sinto-me motivado a continuar o trabalho remotamente (à } \\
\text { distância). }\end{array}$ & & & $-0,302$ & \\
\hline $\begin{array}{l}\text { 6. Interesso-me em participar de cursos de formação para atuar } \\
\text { na Educação à Distância. }\end{array}$ & & & & 0,793 \\
\hline $\begin{array}{l}\text { 7. Interesso-me por participar de fóruns/grupos de discussão com } \\
\text { os colegas sobre assuntos especificamente de trabalho. }\end{array}$ & & & & 0,667 \\
\hline $\begin{array}{l}\text { 5. Acredito que as redes sociais podem ser utilizadas para que } \\
\text { possamos compartilhar informações com os discentes e } \\
\text { servidores }\end{array}$ & & & & 0,328 \\
\hline Quantidade de Itens & 4 & 3 & 3 & 3 \\
\hline Autovalor & 3,991 & 1,756 & 1,505 & 1,260 \\
\hline Variância Explicada (\%) & & 38 , & & \\
\hline Consistência Interna (Alfa de Cronbach) & 0,671 & 0,704 & 0,717 & 0,630 \\
\hline
\end{tabular}

Fonte: Autores.

Conforme apresentado na Tabela 2, a AFE mostrou que os itens se agruparam em 4 grandes fatores, a saber:

Fator 1 - Crenças sobre as dificuldades da Educação Remota Emergencial (4 itens). Este fator representa as convicções que os docentes possuem frente ao ensino remoto (à distância) e a expectativa de retorno das atividades letivas por meio de aulas remotas. O Fator 1 abarca itens, como "sinto que eu tenho dificuldade na realização das minhas atividades docentes de forma remota" e "tenho dúvidas sobre a efetividade de avaliações (testes / provas) ou acompanhamento nas atividades à distância (on-line)".

Fator 2 - Experiência com Ambientes Virtuais de Aprendizagem (3 itens). Este fator representa o grau de experiência, como discente, docente ou tutor em ambientes virtuais de aprendizagem (AVA), abarcando itens como "tenho experiência 
como docente em Ambientes Virtuais de Aprendizagem (AVA)" e "tenho experiência como tutor em Ambientes Virtuais de Aprendizagem (AVA)".

Fator 3 - Desmotivação associada ao ambiente domiciliar (3 itens). Este fator representa as adversidades que os docentes enfrentam para a realização do trabalho remoto em virtude do ambiente domiciliar que podem inibir as condições de trabalho, abarcando itens como "o ambiente em casa não me permite ter concentração para realizar trabalhos remotos" e "meus compromissos domésticos ou familiares me impedem de realizar quaisquer outras atividades”.

4 - Interesse por Atividades Remotas (3 itens). Este fator representa a disponibilidade e a aspiração dos docentes em relação ao trabalho remoto durante o período de distanciamento social. Basicamente, avalia os seus interesses e motivações pessoais dos docentes, abarcando itens, como "interesso-me em participar de cursos de formação para atuar na Educação à Distância" e "acredito que as redes sociais podem ser utilizadas para que possamos compartilhar informações com os discentes e servidores".

\subsection{Capacidades Docentes Inovadoras}

Nesta seção, apresentam-se os resultados descritivos da Escala de Capacidades Educacionais Inovadoras (ECEI), na Tabela 3. Conforme os resultados ressaltam, os docentes se consideram acessíveis aos alunos (81,5\%), mantendo-se atualizados em relação às inovações educacionais (66,7\%), além de utilizar o quadro branco (62,2\%) e os recursos audiovisuais com proficiência $(59,4 \%)$, incluindo a atuação com base nos fatores de aprendizagem (56,5\%) e nas necessidades dos estudantes (55,3\%). Apesar disso, destaca-se que 51,2\% dos docentes afirmaram que a Instituição em que leciona não oferece condições técnicas, materiais e de infraestrutura para o trabalho com Metodologias Ativas, bem como não costumam fazer uso de técnicas de ensino híbrido, combinando aprendizado online com presencial (54,9\%).

Tabela 3: Crenças dos Docentes sobre Capacidades de Ensino Inovadoras.

\begin{tabular}{|c|c|c|c|c|c|}
\hline \multirow{2}{*}{ Itens } & \multirow{2}{*}{ Média } & \multirow{2}{*}{ DP } & \multicolumn{3}{|c|}{ Endosso ao item } \\
\hline & & & Baixo & Médio & Alto \\
\hline $\begin{array}{l}\text { Considero-me um professor acessível aos alunos (telefone, e-mail, } \\
\text { whatsapp, outros). }\end{array}$ & 4,32 & 1,030 & $7,7 \%$ & $10,9 \%$ & $81,5 \%$ \\
\hline $\begin{array}{l}\text { Mantenho-me informado sobre orientações educacionais mais } \\
\text { atualizadas. }\end{array}$ & 3,86 & 0,976 & $8,8 \%$ & $24,5 \%$ & $66,7 \%$ \\
\hline Em sala de aula, costumo utilizar o quadro branco proficuamente. & 3,78 & 1,117 & $15,0 \%$ & $22,8 \%$ & $62,2 \%$ \\
\hline $\begin{array}{l}\text { Em sala de aula, costumo utilizar materiais de áudio/vídeo } \\
\text { proficuamente. }\end{array}$ & 3,73 & 1,174 & $15,6 \%$ & $25,0 \%$ & $59,4 \%$ \\
\hline $\begin{array}{l}\text { Baseio minhas metodologias de ensino nos estilos de aprendizagem } \\
\text { dos discentes. }\end{array}$ & 3,65 & 0,975 & $10,0 \%$ & $33,5 \%$ & $56,5 \%$ \\
\hline $\begin{array}{l}\text { Disponibilizo previamente o texto ou material de apoio para os } \\
\text { discentes. }\end{array}$ & 3,60 & 1,238 & $19,9 \%$ & $24,8 \%$ & $55,3 \%$ \\
\hline Faço uso de metodologias ativas em minhas aulas presenciais. & 3,38 & 1,166 & $21,6 \%$ & $30,1 \%$ & $48,3 \%$ \\
\hline $\begin{array}{l}\text { Mantenho relações acadêmicas com os alunos fora do ambiente } \\
\text { escolar por meio de redes sociais. }\end{array}$ & 3,35 & 1,349 & $28,4 \%$ & $20,9 \%$ & $50,7 \%$ \\
\hline Nas minhas aulas presenciais, costumo propor a sala de aula invertida. & 3,27 & 1,217 & $25,0 \%$ & $32,1 \%$ & $42,9 \%$ \\
\hline $\begin{array}{l}\text { Tenho conhecimento de alguma(s) metodologia(s) inovadora(s) de } \\
\text { ensino relacionadas às metodologias digitais de informação e } \\
\text { comunicação. }\end{array}$ & 3,10 & 1,268 & $33,2 \%$ & $27,4 \%$ & $39,5 \%$ \\
\hline $\begin{array}{l}\text { Utilizo grupos de trabalho remoto em minhas aulas para otimizar o } \\
\text { conteúdo da disciplina. }\end{array}$ & 2,86 & 1,344 & $41,8 \%$ & $24,1 \%$ & $34,0 \%$ \\
\hline $\begin{array}{l}\text { A Instituição em que eu leciono oferece condições técnicas, materiais } \\
\text { e de infraestrutura para o trabalho com Metodologias Ativas. }\end{array}$ & 2,52 & 1,219 & $51,2 \%$ & $28,2 \%$ & $20,6 \%$ \\
\hline $\begin{array}{l}\text { Costumo fazer uso de técnicas de ensino híbrido (metodologia que } \\
\text { combina aprendizado online com presencial). }\end{array}$ & 2,52 & 1,289 & $54,9 \%$ & $21,1 \%$ & $24,0 \%$ \\
\hline
\end{tabular}


Dada à necessidade de compreender o conjunto de itens da ECEI de modo parcimonioso e integrado, utilizou-se a análise fatorial exploratória (AFE). Verificada a adequação dos dados e a fatoriabilidade da matriz de correlações dos itens por meio das estatísticas de Kaiser-Meyer-Olkin $(\mathrm{KMO})=0,835$ e do Teste de Esfericidade de Bartlett, $\chi^{2}(78)=1872,113 ; \mathrm{p}=$ 0,000; procedeu-se à AFE, utilizando o método de extração de máxima verossimilhança e rotação Varimax com normatização de Kaiser, convergida em 5 iterações. Foram extraídos 3 (três) fatores (Tabela 4), com uma explicação de 40,01\% da variância total e cargas fatoriais que variaram de $|0,304|$ a $|0,968|$.

Tabela 4: Itens e Fatores da ECEI.

\begin{tabular}{|c|c|c|c|}
\hline \multirow{2}{*}{ Itens } & \multicolumn{3}{|c|}{ Fatores } \\
\hline & 1 & 2 & 3 \\
\hline $\begin{array}{l}\text { ostumo fazer uso de técnicas de ensino híbrido (metodologia que combina } \\
\text { orendizado online com presencial). }\end{array}$ & 0,927 & & \\
\hline $\begin{array}{l}\text { Tenho conhecimento de alguma(s) metodologia(s) inovadora(s) de ensino } \\
\text { relacionadas às metodologias digitais de informação e comunicação. }\end{array}$ & 0,545 & & \\
\hline Faço uso de metodologias ativas em minhas aulas presenciais. & 0,529 & & \\
\hline $\begin{array}{l}\text { Utilizo grupos de trabalho remoto em minhas aulas para otimizar o conteúdo da } \\
\text { disciplina. }\end{array}$ & 0,494 & & \\
\hline Mantenho-me informado sobre orientações educacionais mais atualizadas. & & 0,641 & \\
\hline Nas minhas aulas presenciais, costumo propor a sala de aula invertida. & & 0,536 & \\
\hline $\begin{array}{l}\text { Baseio minhas metodologias de ensino nos estilos de aprendizagem dos } \\
\text { discentes. }\end{array}$ & & 0,520 & \\
\hline $\begin{array}{l}\text { Considero-me um professor acessível aos alunos (telefone, e-mail, whatsapp, } \\
\text { outros). }\end{array}$ & & & 0,968 \\
\hline $\begin{array}{l}\text { Mantenho relações acadêmicas com os alunos fora do ambiente escolar por } \\
\text { meio de redes sociais. }\end{array}$ & & & 0,479 \\
\hline Em sala de aula, costumo utilizar materiais de áudio/vídeo proficuamente. & & & 0,318 \\
\hline Disponibilizo previamente o texto ou material de apoio para os discentes. & & & 0,304 \\
\hline Quantidade de Itens & 4 & 3 & 4 \\
\hline Autovalor & 4,216 & 1,262 & 1,083 \\
\hline Variância Explicada (\%) & & $40,01 \%$ & \\
\hline Consistência Interna (Alfa de Cronbach) & 0,770 & 0,661 & 0,627 \\
\hline
\end{tabular}

Fonte: Autores.

Conforme apresentado na Tabela 4, a AFE mostrou que os itens se agruparam em 3 grandes fatores, a saber:

Fator 1 - Utilização Corrente de Metodologias Ativas (4 itens). Este fator representa a utilização consciente e corrente por parte do docente de práticas didático-pedagógicas de metodologias ativas com o uso de TDICs na sala de aula presencial, previamente ao período da pandemia. Este fator abarca itens, como "costumo fazer uso de técnicas de ensino híbrido (metodologia que combina aprendizado online com presencial)" e "tenho conhecimento de alguma(s) metodologia(s) inovadora(s) de ensino relacionadas às metodologias digitais de informação e comunicação".

Fator 2 - Práticas Docentes Atualizadas (3 itens). Este fator representa o uso de práticas docentes atualizadas que comungam com necessidades pedagógicas emergentes, abarcando itens como "mantenho-me informado sobre orientações educacionais mais atualizadas" e "baseio minhas metodologias de ensino nos estilos de aprendizagem dos discentes".

Fator 3 - Acessibilidade Docente ao Estudante (4 itens). Este fator representa as relações de proximidade acadêmica e extra-classe entre professores e alunos, considerando a acessibilidade docente ao estudante frente ao cotidiano escolar, abarcando itens como "considero-me um professor acessível aos alunos (telefone, e-mail, WhatsApp, outros)" e "mantenho relações acadêmicas com os alunos fora do ambiente escolar por meio de redes sociais". 


\subsection{Acesso, habilidades e capacidade técnica dos docentes com TDICs}

Nesta seção, apresentam-se os resultados do Questionário de Condições para o Uso de Tecnologias Digitais de Informação e Comunicação (QCU-TDIC), na Tabela 5. Os resultados mostram que a grande maioria dos docentes possui acesso à internet e a equipamentos, o que inclui disponibilidade e condições para a realização de atividades remotas. Destacase que $96,6 \%$ alegam ter, pelo menos, 1 hora por dia de disponibilidade para realizar atividades remotas e 98,5\% têm a acesso à internet (destes, apenas 14,8\% alegaram ter dificuldades de conexão). Também, os dados sobre acesso a equipamentos digitais e computacionais se mostram promissores entre os professores, uma vez que apenas 4,6\% alegaram não ter smartphone e 3,6\% alegaram não ter computador.

Tabela 5: Acesso, Habilidades e Capacidade Técnica com as TDICs.




Nota. ${ }^{1}$ Editor de texto, planilha, apresentação etc. ${ }^{2}$ Impressora, áudio/vídeo, memória disponível. Fonte: Autores.

Em complemento, visando compreender a estrutura de componente do QCU-TDIC, procedeu-se à análise de componentes principais, depois de verificada a adequação dos dados e a fatoriabilidade da matriz de correlações dos itens do questionário por meio das estatísticas de Kaiser-Meyer-Olkin $(\mathrm{KMO})=0,777$ e do Teste de Esfericidade de Bartlett, $\chi^{2}(36)=$ 1639,650; p = 0,000. Extraiu-se um componente, com uma explicação de 40,190\% da variância total e cargas fatoriais que variaram de $|0,453|$ a $|0,721|$, demonstrando a unifatoriabilidade do instrumento, o que indica que todos os itens em conjunto determinam acesso, habilidades e capacidade técnica com as TDICs.

\subsection{Habilidades dos docentes com aplicativos de comunicação, compartilhamento e videoconferência}

Nesta seção, apresentam-se os resultados do Questionário de Habilidades com Aplicativos de Comunicação, Compartilhamento e Videoconferência (QHA-7), na Tabela 6. Os resultados mostram que se predominam as habilidades com os aplicativos mais usuais como o Whatsapp, o Google Drive, e o Skype, e de modo mais disperso o Google Classroom e o Hangouts Meets. Apesar disso, vale ressaltar que a quantidade de professores que não conhecem as ferramentas (especialmente aquelas que são mais cotados para o uso na ERE, como o Google Classroom, o Hangouts Meets, o Zoom e o Google Drive) não é irrisória - devendo se atentar para a necessidade de capacitação a esses tipos de plataforma.

Tabela 6: Habilidades com aplicativos de comunicação, compartilhamento e videoconferência.

\begin{tabular}{cccccc}
\hline & $\begin{array}{c}\text { Não } \\
\text { conhece }\end{array}$ & $\begin{array}{c}\text { Conhece, mas } \\
\text { possui pouca } \\
\text { habilidade }\end{array}$ & $\begin{array}{c}\text { Conhece e possui } \\
\text { habilidade } \\
\text { mediana }\end{array}$ & $\begin{array}{c}\text { Conhece e possui } \\
\text { habilidade } \\
\text { substacial }\end{array}$ & $\begin{array}{c}\text { Conhece e possui } \\
\text { expertise ou } \\
\text { grande habilidade }\end{array}$ \\
\cline { 2 - 5 } $\begin{array}{c}\text { Drive } \\
\text { Google } \\
\text { Classroom } \\
\text { Whatsapp }\end{array}$ & $5,3 \%$ & $21,6 \%$ & $27,2 \%$ & $31,1 \%$ & $14,8 \%$ \\
\cline { 2 - 6 } & $18,9 \%$ & $28,4 \%$ & $20,7 \%$ & $22,8 \%$ & $9,2 \%$ \\
\cline { 2 - 5 } $\begin{array}{c}\text { Hangouts } \\
\text { Meets }\end{array}$ & $15,1 \%$ & $20,7 \%$ & $14,1 \%$ & $52,9 \%$ & $30,8 \%$ \\
\cline { 2 - 5 } \begin{tabular}{c} 
Zoom \\
\cline { 2 - 5 }
\end{tabular} & $36,9 \%$ & $24,8 \%$ & $22,4 \%$ & $31,3 \%$ & $10,4 \%$ \\
\cline { 2 - 5 } $\begin{array}{c}\text { Skype } \\
\text { Lives }\end{array}$ & $11,4 \%$ & $32,8 \%$ & $18,4 \%$ & $15,0 \%$ & $4,9 \%$ \\
\cline { 2 - 5 } $\begin{array}{c}\text { instagram / } \\
\text { Youtube) }\end{array}$ & $21,1 \%$ & $42,3 \%$ & $24,7 \%$ & $23,5 \%$ & $4,7 \%$ \\
\hline
\end{tabular}

Fonte: Autores.

Em complemento, visando compreender a estrutura de componente do QHA-7, procedeu-se à análise de componentes principais, depois de verificada a adequação dos dados e a fatoriabilidade da matriz de correlações dos itens do questionário por meio das estatísticas de Kaiser-Meyer-Olkin $(\mathrm{KMO})=0,882$ e do Teste de Esfericidade de Bartlett, $\chi^{2}(21)=1832,333 ; \mathrm{p}=$ 0,000. Foi extraído um componente, com uma explicação de 55,626\% da variância total e cargas fatoriais que variaram de |0,583| a |0,849|, garantindo-se a significância prática de explicação e demonstrando a unifatoriabilidade do instrumento, o que indica que todos os itens da QHA-7 em conjunto podem determinar as habilidades com aplicativos de comunicação, compartilhamento e videoconferência. 


\subsection{Testagem dos modelos preditivos}

Após as análises pormenorizadas dos instrumentos e questionários aplicados, ajuizou-se verificar relações preditivas entre os fatores e componentes que foram extraídos por meio da análise fatorial. Tendo-se os fatores, planejou-se testar as dimensões avaliadas nos instrumentos ECEI, QCU-TDIC e QHA-7 como preditores dos aspectos relacionados ao trabalho docente remoto em virtude da pandemia da COVID-19 (avaliados na ERP-TRD). Apesar disso, a ERP-TRD apresentou em seus Fatores 2 e 3, dimensões exógenas ao trabalho remoto e, portanto, ao invés de serem tratados como variáveis consequentes (dependentes), estes fatores foram incluídos nas análises como variáveis preditoras (independentes).

Assim, foram estabelecidos dois modelos hipotético-teóricos de explicação para as Crenças sobre as dificuldades da Educação Remota Emergencial (Fator 1 da ERP-TRD) e o Interesse por Atividades Remotas (Fator 4 da ERP-TRD), em que as seguintes variáveis foram estabelecidas como variáveis independentes:

- Experiência com Ambientes Virtuais de Aprendizagem (Fator 2 da ERP-TRD).

- Desmotivação associada ao ambiente domiciliar (Fator 3 da ERP-TRD).

- Utilização Corrente de Metodologias Ativas (Fator 1 da ECEI).

- Práticas Docentes Atualizadas (Fator 2 da ECEI).

- Acessibilidade Docente ao Estudante (Fator 3 da ECEI).

- Acesso, Habilidades e Capacidade Técnica com as TDICs (Fator único do QCU-TDIC).

- Habilidades com aplicativos de comunicação, compartilhamento e videoconferência (Fator único do QHA-7).

Nas Tabelas 7 e 8, apresentam-se os modelos testados. Para o modelo de regressão predizendo as Crenças sobre as dificuldades da Educação Remota Emergencial (Fator 1 da ERP-TRD), na Tabela 7, a desmotivação associada ao ambiente domiciliar (Fator 3 da ERP-TRD), a utilização corrente de metodologias ativas (Fator 1 da ECEI) e a experiência com ambientes virtuais de aprendizagem (Fator 2 da ERP-TRD) demonstraram maior relação preditiva.

Tabela 7: Modelo de Regressão predizendo as Crenças sobre as dificuldades da Educação Remota Emergencial (Fator 1 da ERP-TRD).

\begin{tabular}{|c|c|c|c|c|c|c|}
\hline Variáveis Preditoras & $\mathrm{r}$ de Pearson & $\mathrm{B}$ & EP & Beta & $\mathrm{T}$ & $p$ \\
\hline Constante & & 3,620 & 0,295 & & 12,290 & 0,000 \\
\hline $\begin{array}{l}\text { Desmotivação associada ao ambiente } \\
\text { domiciliar }\end{array}$ & $0,532(0,000)$ & 0,389 & 0,033 & 0,395 & 11,660 & 0,000 \\
\hline $\begin{array}{l}\text { Utilização Corrente de Metodologias } \\
\text { Ativas }\end{array}$ & $-0,463(0,000)$ & $-0,231$ & 0,042 & $-0,228$ & $-5,530$ & 0,000 \\
\hline $\begin{array}{l}\text { Experiência com Ambientes Virtuais } \\
\text { de Aprendizagem }\end{array}$ & $-0,422(0,000)$ & $-0,141$ & 0,029 & $-0,177$ & $-4,816$ & 0,000 \\
\hline $\begin{array}{l}\text { Acesso, Habilidades e Capacidade } \\
\text { Técnica com as TDICs }\end{array}$ & $-0,462(0,000)$ & $-0,214$ & 0,086 & $-0,112$ & $-2,479$ & 0,013 \\
\hline Acessibilidade Docente ao Estudante & $-0,205(0,000)$ & 0,148 & 0,043 & 0,124 & 3,429 & 0,001 \\
\hline $\begin{array}{l}\text { Habilidades com aplicativos de } \\
\text { comunicação... }\end{array}$ & $-0,425(0,000)$ & $-0,108$ & 0,054 & $-0,091$ & $-2,010$ & 0,045 \\
\hline & & & \multicolumn{4}{|c|}{$r=0,675$} \\
\hline & & & \multicolumn{4}{|c|}{$r^{2}=0,456$} \\
\hline & & & \multirow{2}{*}{\multicolumn{4}{|c|}{$\begin{array}{l}\text { Teste Durbin-Watson }=1,800 \\
\mathrm{~F}(6,581)=81053 \cdot n=0.000\end{array}$}} \\
\hline & & & & & & \\
\hline
\end{tabular}

Fonte: Autores.

A relação preditiva positiva entre a desmotivação associada ao ambiente domiciliar (Fator 3 da ERP-TRD) e as crenças sobre as dificuldades da Educação Remota Emergencial (Fator 1 da ERP-TRD), mostra que quanto maiores os 
intervenientes presentes no cotidiano domiciliar do docente (p.ex., demandas familiares e atividades domésticas), mais fortemente é ressaltada as dificuldades em relação às aulas remotas (p.ex., incertezas sobre a efetividade do processo de ERE e o desejo de não retorno às atividades letivas remotas).

Conjuntamente, a relação preditiva negativa da utilização corrente de metodologias ativas (Fator 1 da ECEI) e da experiência com ambientes virtuais de aprendizagem (Fator 2 da ERP-TRD) frente às crenças sobre as dificuldades da Educação Remota Emergencial (Fator 1 da ERP-TRD) corroboram essa linha de pensamento. Complementa-se, portanto, que não apenas a desmotivação é um fator mediador das incertezas e do baixo endosso às aulas remotas, mas a falta de conhecimentos de metodologias ativas e de experiências com AVAs.

Para o modelo de regressão predizendo o Interesse por Atividades Remotas (Fator 4 da ERP-TRD), na Tabela 8, a desmotivação associada ao ambiente domiciliar (Fator 3 da ERP-TRD), as práticas docentes atualizadas (Fator 2 da ECEI) e a acessibilidade docente ao estudante (Fator 3 da ECEI) demonstraram maior relação preditiva.

Tabela 8: Modelo de Regressão predizendo o Interesse por Atividades Remotas (Fator 4 da ERP-TRD).

\begin{tabular}{|c|c|c|c|c|c|c|}
\hline Variáveis Preditoras & r de Pearson & B & EP & Beta & $\mathrm{T}$ & $P$ \\
\hline Constante & & 2,911 & 0,321 & & 9,062 & 0,000 \\
\hline $\begin{array}{l}\text { Desmotivação associada ao ambiente } \\
\text { domiciliar }\end{array}$ & $-0,338(0,000)$ & $-0,201$ & 0,036 & $-0,235$ & $-5,641$ & 0,000 \\
\hline Práticas Docentes Atualizadas & $0,271(0,000)$ & 0,154 & 0,044 & 0,147 & 3,514 & 0,000 \\
\hline Acessibilidade Docente ao Estudante & $0,275(0,000)$ & 0,119 & 0,046 & 0,115 & 2,599 & 0,010 \\
\hline $\begin{array}{l}\text { Acesso, Habilidades e Capacidade } \\
\text { Técnica com as TDICs }\end{array}$ & $0,282(0,000)$ & 0,176 & 0,073 & 0,106 & 2,416 & 0,016 \\
\hline & & & \multicolumn{4}{|c|}{$r=0,425$} \\
\hline & & & \multicolumn{4}{|c|}{$\mathrm{r}^{2}=0,180$} \\
\hline & & & \multicolumn{4}{|c|}{$r^{2}$ ajustado $=0,175$} \\
\hline & & & \multicolumn{4}{|c|}{ Teste Durbin-Watson $=1,904$} \\
\hline & & & \multicolumn{4}{|c|}{$\mathrm{F}(4,583)=32,087 ; p=0,000$} \\
\hline
\end{tabular}

Fonte: Autores.

A relação preditiva negativa entre a desmotivação associada ao ambiente domiciliar (Fator 3 da ERP-TRD) e o interesse por atividades remotas (Fator 4 da ERP-TRD), mostra que quanto menor a sensação de desmotivação por parte do docente - especialmente em virtude das questões domiciliares associadas ao distanciamento social, incluindo demandas familiares e atividades domésticas -, maior é o interesse em realizar atividades administrativas e letivas remotamente, corroborando a análise relacionada às crenças sobre dificuldades da ERE.

O modelo de regressão apresenta, também, relações preditivas positivas (fracas) das práticas docentes atualizadas (Fator 2 da ECEI) e da acessibilidade docente ao estudante (Fator 3 da ECEI) com o interesse por atividades remotas (Fator 4 da ERPTRD). Isso sugere que o interesse dos professores por atividades remotas estaria vinculado a características pessoais dos docentes.

\section{Discussão}

Inicialmente, vale destacar que os resultados mostraram que a maioria dos professores investigados apresentou predisposição e motivação em realizar atividades remotas, endossando o interesse por participar de cursos de capacitação e momentos de discussão sobre as possibilidades de aulas remotas, incluindo a propensão do uso das redes sociais para aproximação com os estudantes. Sobre este aspecto, os professores mostraram-se acessíveis aos estudantes e alegaram se utilizar de práticas docentes atualizadas.

Apesar disso, ressalta-se que os professores investigados demonstraram ter dúvidas sobre a efetividade de avaliações 
(testes/provas) nesse modelo de aulas remotas, bem como, alegaram que provavelmente terão dificuldades na realização de atividades remotas, especialmente aquelas relacionadas ao ensino e ao acompanhamento dos estudantes.

A análise de regressão endossou essa linha de raciocínio. O interesse pelas aulas remotas demonstrou relação com uma menor quantidade de demandas domésticas e domiciliares, o uso de práticas docentes atualizadas, a acessibilidade do docente ao estudante e o profícuo a acesso e uso da internet e de equipamentos digitais e computacionais.

Especificamente sobre o acesso, as habilidades e a capacidade técnica com as TDICs, a grande maioria dos docentes demonstrou condições favoráveis de acesso e uso. Apesar disso, aqueles com maiores dificuldades de acesso e uso requerem uma atenção especial, pois são justamente aqueles com menores condições de retorno às atividades letivas remotas. A análise de regressão ressalta essa relação preditiva, indicando que as dificuldades da ERE estão relacionadas mais fortemente a uma maior desmotivação associada ao ambiente domiciliar, à falta de experiência com metodologias ativas e ambientes virtuais de aprendizagem e às dificuldades de acesso e uso de TDICs.

Este estudo mostra também que a desmotivação e a influência do ambiente domiciliar estão interrelacionadas formando o Fator 3 da ERP-TRD. Conforme trazido no referencial teórico, o trabalho remoto realizado no ambiente domiciliar, especialmente no cenário de pandemia, traz uma redução de produtividade, visto que intervenientes do ambiente domiciliar podem diminuir a concentração ou se estabelecer como demandas e necessidades imediatas. Além disso, quando se tratam de atividades letivas associadas a demandas administrativas, a elevação dos níveis de estresse e ansiedade pode trazer implicações adversas para a saúde mental do professor - Taylor (2019) alerta para as ocorrências de sofrimento emocional e comportamentos desadaptativos, devido a questões como medo, trauma, luto, problemas financeiros etc.

Por sua vez, a análise das habilidades com aplicativos de comunicação, compartilhamento e videoconferência mostram que parte dos docentes não possui conhecimentos sobre uso das principais ferramentas que são comumente relacionadas à ERE (p.ex., Google Classroom, Hangouts Meets, Zoom, Google Drive). Os resultados ressaltam, portanto, a necessidade de realização de cursos de capacitação e treinamentos dos docentes para uso dessas ferramentas, dirimindo também as incertezas sobre a efetividade do processo de ERE e aumento o interesse e as condições de retorno às atividades letivas remotamente.

\section{Considerações Finais}

Este trabalho traça um panorama inicial sobre condições, expectativas e capacidades de professores de cursos técnicos e superiores presenciais de instituições públicas e privadas de ensino brasileiras quanto à Educação Remota Emergencial (ERE), em virtude do distanciamento social associado à pandemia da COVID-19. Este estudo, portanto, contribui para ações da gestão escolar no Brasil quanto à retomada das atividades letivas durante o período de distanciamento social.

O estudo revela que os docentes, de modo geral, apresentam condições e interesse para a realização de atividades letivas remotas, embora apresentem dúvidas e receios sobre a efetividade das atividades remotas. Assim, compreende-se a necessidade de encarar a ERE de modo ponderado e parcimonioso, visto que aqueles docentes com dificuldades de acesso e uso de TDICs e aqueles com dificuldades associadas ao ambiente domiciliar irão exigir gerenciamento adequado e bom senso.

As limitações deste estudo recaem, especialmente, sobre a temporalidade dos resultados apresentados - coletados entre 19 e 30 de junho de 2020 -, que representam uma realidade em transição, podendo se agravar (em virtude do adoecimento psicológico ou contágio com COVID-19) ou se atenuar (em virtude do recebimento de auxílios, realização de cursos de capacitação ou surgimento de uma vacina). O tipo de pesquisa com tratamento amostral não-probabilístico também é uma limitação deste estudo, pois reduz a possibilidade generalização dos resultados aqui encontrados. Portanto, sugere-se que novos estudos investiguem questões específicas sobre o trabalho docente neste período pandêmico, aprofundando e discutindo os resultados das pesquisas até então realizadas. 


\section{Agradecimentos}

Agradecemos ao Instituto Federal do Norte de Minas Gerais (IFNMG) pelo apoio institucional dado ao Projeto que deu origem a este artigo.

\section{Referências}

Arruda, E. P. (2020). Educação remota emergencial: elementos para políticas públicas na educação brasileira em tempos de Covid-19. EmRede - Revista de Educação à Distância, 7(1), 257-275.

Anderson, R. M., Heesterbeek, H., Klinkenberg, D., \& Hollingsworth, T. D. (2020). How will country-based mitigation measures influence the course of the COVID-19 epidemic? The Lancet, 95(10228), 931-934. doi: 10.1016/S0140-6736(20)30567-5

Azevedo, D. S., Silveira, A. C., Lopes, C. O., Amaral, L. de O., Goulart, I. do C. V., \& Martins, R. X. (2018). Letramento digital: uma reflexão sobre o mito dos "Nativos Digitais". RENOTE - Revista Novas Tecnologias na Educação, 16(2), 615-625.

Barreto, A. C. F., \& Rocha, D. S. (2020). COVID 19 e educação: resistências, desafios e (im)possibilidades. Revista Encantar - Educação, Cultura e Sociedade, 2, 1-15. doi: 10.46375/encantar. v2.0011

Barros, A. M., \& Silva, J. R. G. D. (2010). Percepções dos indivíduos sobre as consequências do teletrabalho na configuração home-office: estudo de caso na Shell Brasil. Cadernos EBAPE.BR, 8(1), 71-91. doi: 10.1590/S1679-39512010000100006

Bathini, D. R., \& Kandathil, G. M. (2019). An orchestrated negotiated exchange: Trading home-based telework for intensified work. Journal of Business Ethics, 154(2), 411-423. doi: 10.1007/s10551-017-3449-y

Bedford, J., Enria, D., Giesecke, J., Heymann, D. L., Ihekweazu, C., ... Wieler, L. H. (2020). COVID-19: towards controlling of a pandemic. The Lancet, 395(10229), 1015-1018. doi: 10.1016/S0140-6736(20)30673-5

Beluce, A. C., \& Oliveira, K. L. (2016). Escala de estratégias e motivação para aprendizagem em ambientes virtuais. Revista Brasileira de Educação, 21(66), 593-610.

Brasil. Ministério da Educação. Gabinete do Ministro. (2020a). Portaria nº 343, de 17 de março de 2020. Dispõe sobre a substituição das aulas presenciais por aulas em meios digitais enquanto durar a situação de pandemia do Novo Coronavírus - COVID-19. Diário Oficial da União, Brasília, DF, p. 1.

Brasil. Ministério da Educação. Gabinete do Ministro. (2020b). Portaria n ${ }^{o} 345$, de 19 de março de 2020. Altera a Portaria MEC no 343 , de 17 de março de 2020 que dispõe sobre a substituição das aulas presenciais por aulas em meios digitais enquanto durar a situação de pandemia do Novo Coronavírus - COVID19. Diário Oficial da União, Brasília, DF.

Brasil. Ministério da Educação. Gabinete do Ministro. (2020c). Portaria $n^{o} 376$, de 3 de abril de 2020. Dispõe sobre as aulas nos cursos de educação profissional técnica de nível médio, enquanto durar a situação de pandemia do novo coronavírus - Covid-19. Diário Oficial da União, Brasília, DF, p. 66.

Brasil. Ministério da Educação. Gabinete do Ministro. (2020d). Portaria $n^{\circ} 395$, de 15 de abril de 2020 . Prorroga o prazo previsto no $§ 1^{\circ}$ do art. $1^{\circ}$ da Portaria n 343, de 17 de março de 2020. Diário Oficial da União, Brasília, DF, p. 61.

Brasil. Ministério da Educação. (2020e). Portal: Coronavírus - Monitoramento das Instituições de Ensino. MEC: Brasília. Obtido em http://portal.mec.gov.br/coronavirus/

Camacho, A. C. L. F., Joaquim, F. L., Menezes, H. F., \& Sant'Anna, R. M. (2020). A tutoria na educação à distância em tempos de COVID-19: orientações relevantes. Research, Society and Development, 9(5), e30953151. doi: 10.33448/rsd-v9i5.3151 
Castaman, A. S., \& Rodrigues, R. A. (2020). Educação a Distância na crise COVID-19: um relato de experiência. Research, Society and Development, 9(6), e180963699. doi: 10.33448/rsd-v9i6.3699

Coelho, W. G., \& Tedesco, P. C. de A. R. (2017). A percepção do outro no ambiente virtual de aprendizagem: presença social e suas implicações para Educação a Distância. Revista Brasileira de Educação, 22(70), 609-624.

Christensen, K. (1988). The new era of home-based work: directions and policies. New York, NY: Routledge.

Freitas, M. T. (2010). Letramento digital e formação de professores. Educação em Revista, 26(3), 335-352.

Gaudecker, H. M. V., Holler, R., Janys, L., Siflinger, B., \& Zimpelmann, C. (2020). Labour supply in the early stages of the CoViD-19 pandemic: empirical evidence on hours, home office, and expectations. Institute of Labor Economics (IZA), Discussion Papers, $\mathrm{n}^{\circ}$ 13158. Obtido em http://ftp.iza.org/dp13158.pdf

Gomes, V. T. S., Rodrigues, R. O., Gomes, R. N. S., Gomes, M. S., Viana, L. V. M., \& Silva, F. S. (2020). A Pandemia da Covid-19: Repercussões do Ensino Remoto na Formação Médica. Revista Brasileira de Educação Médica, 44(4), e-114. doi: 10.1590/1981-5271v44.4-20200258

Joye, C. R., Moreira, M. M., \& Rocha, S. S. D. (2020). Distance education or emergency remote educational activity: in search of the missing link of school education in times of COVID-19. Research, Society and Development, 9(7), e521974299-e521974299. doi: 10.33448/rsd-v9i7.4299

Kaieski, N., Grings, J. A., \& Fetter, S. A. (2015). Um estudo sobre as possibilidades pedagógicas de utilização do Whatsapp. RENOTE - Revista Novas Tecnologias na Educação, 13(2), 1-10. doi: 10.22456/1679-1916.61411

Kissler, S. M., Tedijanto, C., Goldstein, E., Grad, Y. H., \& Lipsitch, M. (2020). Projecting the transmission dynamics of SARS-CoV-2 through the postpandemic period. Science, eabb5793. doi: 10.1126/science. abb5793

Macedo, Y. M., Ornellas, J. L., \& Bomfim, H. F. (2020). COVID-19 no Brasil: o que se espera para população subalternizada? Revista Encantar - Educação, Cultura e Sociedade, 2, 1-10. doi: 10.5935/encantar. v2.0001

Maia, C., \& Mattar, J. (2008). ABC da EaD: a educação a distância hoje. São Paulo: Pearson Prentice Hall.

Mill, D. (2012). Docência virtual: uma visão crítica. Campinas: Papirus.

Nunes, A. K. F., Oliveira, A. V. B., \& Sabino, R. F. (2019). Docência na educação a distância: abordagem sobre o perfil professional. Revista Internacional de Educação Superior, 5, e019009, 1-16.

Peeri, N. C., Shrestha, N., Rahman, S., Zaki, R., Tan, Z., ... Haque, U. (2020). The SARS, MERS and novel coronavirus (COVID-19) epidemics, the newest and biggest global health threats: what lessons have we learned? International Journal of Epidemiology, 1-10, In Press. doi: 10.1093/ije/dyaa033

Pereira, A. S., Shitsuka, D. M., Parreira, F. J., \& Shitsuka, R. (2018). Metodologia da pesquisa científica. Santa Maria: UAB/NTE/UFSM.

Remuzzi, A., \& Remuzzi, G. (2020). COVID-19 and Italy: what next? The Lancet, In Press. doi: 10.1016/S0140-6736(20)30627-9

Sanchez-Taltavull, D., Candinas, D., Roldán, É., \& Beldi, G. (2020). Modelling strategies to organize healthcare workforce during pandemics: application to COVID-19. medRxiv, In Press. doi: 10.1101/2020.03.23.20041863

Saraiva, K., Traversini, C., \& Lockmann, K. (2020). A educação em tempos de COVID-19: ensino remoto e exaustão docente. Práxis Educativa, 15, e2016289. doi: 10.5212/PraxEduc.v.15.16289.094

Taylor, S. (2019). The psychology of pandemics: Preparing for the next global outbreak of infectious disease. Newcastle upon Tyne: Cambridge Scholars Publishing. 
Research, Society and Development, v. 10, n. 1, e37510111904, 2021

(CC BY 4.0) | ISSN 2525-3409 | DOI: http://dx.doi.org/10.33448/rsd-v10i1.11904

Toquero, C. (2020). M. Emergency remote education experiment amid COVID-19 pandemic. International Journal of Educational Research and Innovation, Covid-19 \& Education Edition, 15, 162-172. doi: 10.46661/ijeri.5113

Viner, R. M., Russel, S. J., Croker, H., Packer, J., Ward, J., ... Booy, R. (2020). School closure and management practices during coronavirus outbreaks including COVID-19: a rapid systematic review. The Lancet Child \& Adolescent Health, 4(5), 397-404. doi: 10.1016/S2352-4642(20)30095-X

World Health Organization (2020). Coronavirus disease (COVID-19) - Weekly Epidemiological Update - 24 August 2020. Obtido em https://www.who.int/docs/default-source/coronaviruse/situation-reports/20200824-weekly-epi-update.pdf?sfvrsn=806986d1_4

Williamson, B., Eynon, R., \& Potter, J. (2020). Pandemic politics, pedagogies and practices: digital technologies and distance education during the coronavirus emergency. Learning, Media and Technology, 45(2), 107-114. doi: 10.1080/17439884.2020.1761641 